\title{
Misclassification of patients with spinocerebellar ataxia as having psychogenic postural instability based on computerized dynamic posturography
}

\author{
Susan J. Herdman ${ }^{1,2,3 *}$, Courtney D. Hall ${ }^{1}$, Rachael Eggers ${ }^{1 \dagger}$, Stasha Sampson ${ }^{1+}$, Sydne Goodier ${ }^{1 \dagger}$ and \\ Becky Filson ${ }^{1+}$
}

1 Rehabilitation Medicine, Emory University, Atlanta, GA, USA

2 Otolaryngology-Head Neck Surgery, Emory University, Atlanta, GA, USA

${ }^{3}$ Rehabilitation Research and Development, Atlanta VA Medical Center, Decatur, GA, USA

\section{Edited by:}

Robert Daroff, Case Western Reserve

University School of Medicine, USA

Michael Strupp,

Ludwig-Maximilians-Universität

München, Germany

\section{Reviewed by:}

Mark F. Walker, Case Western

Reserve University, USA

Douglas Lanska, VA Medical Center, USA

\section{*Correspondence:}

Susan J. Herdman, Department of Rehabilitation Medicine, Emory

University School of Medicine, 1441 Clifton Road NE, Atlanta, GA 30329, USA.

e-mail: sherdma@emory.edu

${ }^{\dagger}$ Research performed as a part of the requirements for the DPT degree.

Specific criteria have been developed based on computerized dynamic posturography (CDP) to assist clinicians in identifying patients with psychogenic balance problems (Cyr and Cevette, 1993; Cevette et al., 1995; Goebel et al., 1997; Gianoli et al., 2000). Patients with known spinocerebellar ataxia (SCA) meet several of the criteria for psychogenic balance problem and risk being misclassified as having imbalance of psychogenic origin. However, our research shows that patients with SCA may be distinguished from patients with psychogenic balance problems in several ways. We compared test performance on CDP and the observation of specific behaviors that are associated with psychogenic balance problems in patients with SCA $(n=43)$ and patients with known psychogenic balance problems $(n=40)$. Chi-square analysis was used to determine if there were significant differences between the groups for the frequency of each criterion for psychogenic CDP and Observed Behaviors. Level of significance was Bonferroni corrected for multiple comparisons. Sensitivity, specificity, and positive likelihood ratios were calculated for each criterion. Hierarchical cluster analysis was used to examine whether the two patient groups demonstrated similar groupings of criteria. Comparison of the results of these analyses identified two criteria that were significantly more frequent in the psychogenic group than in the SCA group: regular periodicity of sway and circular sway. Sensitivity, specificity, and positive likelihood ratios identified two additional criteria, inconsistent motor responses and large lateral sway that also seem to suggest a psychogenic component to a person's imbalance. Prospective studies are needed to validate the usefulness of these findings.

\section{Keywords: spinocerebellar ataxia, pyschogenic imbalance, computerized dynamic posturography}

\section{INTRODUCTION}

An important part of the clinical evaluation of dizziness and imbalance is to distinguish between physiologic and psychogenic causes of postural instability. Specific criteria have been developed based on computerized dynamic posturography (CDP) to assist clinicians in identifying patients with psychogenic balance problems (Cyr and Cevette, 1993; Cevette et al., 1995; Goebel et al., 1997; Gianoli et al., 2000). We noticed that patients with known spinocerebellar ataxia (SCA) appeared to meet several of the criteria for psychogenic balance problem. This is of concern because if patients with a physiological basis for instability are misclassified as having imbalance of psychogenic origin, inappropriate decisions about treatment could be made. The purpose of the study was to: (1) establish if patients with known SCA meet criteria established for diagnosing psychogenic balance problems and (2) determine whether there are different patterns of criteria associated with SCA compared to psychogenic basis for imbalance.

\section{MATERIALS AND METHODS}

\section{SUBJECTS}

Informed consent was obtained in compliance with the Emory University Institutional Review Board protocol.

\section{Patients with SCA}

The most common symptoms associated with SCA are progressive deterioration of balance and cerebellar ataxia (Manto, 2005; Teive, 2009). SCA is a term that encompasses many diagnoses associated with degeneration of the anterior vermis and paravermis of the anterior lobe of the cerebellum. There are currently 30 different forms of SCA some of which have been classified molecularly by their gene descriptions. Only those patients with known SCA documented by a neurologist were included in this category.

Patients with psychogenic balance disorders: Patients with complaints of dizziness plus psychological and/or psychiatric problems were included in this group. Psychological and/or psychiatric problems could include anxiety, depression, panic disorders, conversion and somatoform disorders, fear of falling, treatment for a psychiatric problem in the past, and psychogenic presentation on clinical examination (e.g., give-away weakness, astasia /abasia). For all patients in this group, the identification of a psychological/ psychiatric problem as the basis for their dizziness and the exclusion of a physiologic basis for imbalance were made by a neurologist prior to performance of CDP. 


\section{Exclusion criteria}

Patients were excluded if they had a history of dementia, cerebral vascular accident, lower extremity amputation, abnormal vestibular function tests, a history of peripheral vestibular dysfunction, or any other neurological disorder affecting balance and gait.

\section{MEASUREMENT OF POSTURAL STABILITY}

Postural stability was measured using Computerized Dynamic Posturography (NeuroCom International, Inc., Clackamas, OR, USA). Center of foot pressure was measured by a series of force transducers positioned under a movable support surface. In one set of tests (sensory organization test, SOT), this information is used to move either a visual surround or the support surface in approximate synchrony with the person's sway. This results in altered visual and/or somatosensory feedback (Table 1). Data also include maximum peak-to-peak anterior-posterior (AP) sway, sway path, and center of gravity alignment. Automatic postural responses were assessed by measuring the patient's response to sudden translational or pitch perturbations of the support surface. CDP has been shown to have both construct and content validity (Hall and Herdman, 2005).

\section{CRITERIA FOR DETERMINING THE EXISTENCE OF PSYCHOGENIC CONTRIBUTION TO POSTURAL INSTABILITY}

(1) The calculated SOT scores is highest for aphysiologic score suggesting an aphysiologic basis for test results (Cevette et al., 1995).

(2) Substandard performance on sensory tests (ST) 1 and 2 (Cevette et al., 1995; Goebel et al., 1997; Gianoli et al., 2000): The presence of more than one trial that was below normal (increased sway or loss of balance) for age.

(3) Large amplitude AP sway: Score $=$ the average number of AP sways that exceed $5^{\circ}$ without falls on ST 4, 5, and 6 (Goebel et al., 1997; Gianoli et al., 2000). The subject had to have $>5^{\circ}$

Table 1 | Test conditions for computerized dynamic posturography.

\begin{tabular}{ll}
\hline $\begin{array}{l}\text { Sensory } \\
\text { test }\end{array}$ & Description \\
\hline 1 & Static standing with the eyes open on a fixed, firm surface, \\
& visual, somatosensory, and vestibular inputs accurate \\
& Static standing with the eyes closed on a fixed, firm surface; \\
& somatosensory and vestibular inputs are available while visual \\
& input is removed \\
& Standing on a fixed support while the visual surround sways \\
& anteriorly and posteriorly; somatosensory and vestibular inputs \\
& are available while visual inputs are inaccurate \\
& Standing with the eyes open while the support surface moves; \\
& visual and vestibular inputs are correct while somatosensory \\
& input is inaccurate \\
& Standing with eyes closed to remove visual input while surface \\
& moves to provide inaccurate somatosensory cues, relying on \\
& vestibular system for postural control \\
& Visual surround and support surface are both moving providing \\
& inaccurate visual and somatosensory input, relying on vestibular \\
& system for postural control
\end{tabular}

AP sway on at least two trials in more than one test condition (ST 4-6).

(4) Large amplitude lateral sway: Score $=$ the average number of lateral sways that exceed $1.25^{\circ}$ without falls on ST 4,5 , and 6 (Goebel et al., 1997; Gianoli et al., 2000). The subject had to have $>1.25^{\circ}$ lateral sway on at least one trial of one test conditions ST 4-6.

(5) Better performance on a more difficult test (ST 5 and 6) than on easier tests (ST 1 and 2): this was determined using the formulae $\quad$ Score $=[($ Score $1-$ Norm 1$)+($ Score $2-$ Norm 2$)]-$ $[($ Score $5-$ Norm 5$)+($ Score6 - Norm6 $)]$. We used the average of all three trials for each test in the formulae (Cevette et al., 1995).

(6) Regular periodicity of sway (Mallinson and Longridge, 2005): This was based on qualitative judgment by the rater of sway across all trials.

(7) Circular sway on one or more trials in which the support surface was moving in pitch (Goebel et al., 1997; Krempl and Dobie, 1998; Gianoli et al., 2000; Mallinson and Longridge, 2005).

(8) Excessive inter-trial variability: This was based on the qualitative judgment by the rater of the variability across all test conditions (Cevette et al., 1995; Mallinson and Longridge, 2005).

(9) Inconsistent motor responses to small and large translational perturbations: more than one of the four tests had to exhibit one or more discordant tests to be positive (qualitative judgment by the rater; Goebel et al., 1997).

(10) Observed behaviors that can be psychogenic (Lempert et al., 1991; Table 2): The presence of one or more of the criteria was considered positive.

\section{PROCEDURE}

A retrospective chart review from 2003 to 2007 identified all patients with SCA or with a diagnosis of psychogenic balance problems who had CDP testing. These records were examined to eliminate patients meeting exclusion criteria. All patients had been seen by a neurologist in the Dizziness and Balance Center before being referred for the CDP test. CDP testing was completed by a trained technician as a part of the patient's initial evaluation. A computer-generated summary of the posturography results along with an observation form filled out by the technician were provided to one of two physical therapists (Susan J. Herdman, Courtney D. Hall) blinded to the patient's diagnosis. The therapist then summarized the results and identified which, if any, of the

\section{Table 2 | Clinical behaviors suggestive of a psychogenic balance} problem (Lempert et al., 1991).

\section{Observed behavior}

Moment to moment fluctuations in the patient's level of impairment (s) Excessive slowness or hesitation

Exaggerated sway on Romberg, improved by distraction

Uneconomical postures with waste of muscular energy

Extreme caution with restricted steps (walking on ice

Sudden buckling of the knees, typically without falling 
psychogenic criteria were met. The results of the posturography test, the physical therapist's summary report, and the physician's notes were the basis of the analysis described below.

\section{STATISTICS}

One-way ANOVA was used to identify group differences for age and number of criteria met. Chi-square analysis was used to determine if there were significant differenced between the groups for the frequency of each of the criteria for psychogenic performance and for the number of participants meeting three or fewer, four to five, and more than five criteria. We chose those criteria based on Mallinson and Longridge (2005), who found that participants meeting three or fewer criteria were unlikely to have a psychogenic component to their balance problem, participants meeting four or five criteria possibly had a psychogenic component and participants meeting more than five criteria were likely to have a psychogenic component. Level of significance was Bonferroni corrected for multiple comparisons $(p<0.005)$. Sensitivity, specificity, and likelihood ratios were calculated for each of the CDP criteria. Sensitivity was defined as the ability of CDP or observational criterion to identify a person as psychogenic when they really were. Specificity was defined as the ability of CDP or observational criterion to identify a person as not in the psychogenic category when they really were not, e.g., they were in the SCA group. Positive likelihood ratios describe whether or not a test result (CDP or Observational criteria) changes the probability that a condition (being in the psychogenic group) exists. A Likelihood ratio of $>1$ indicates that the test result is associated with being in the psychogenic group; ratios $<1$ indicates that the result is associated with the absence of the disease (are not in psychogenic group).

Hierarchical cluster analysis (HCA) was used to organize the criteria into groups with similar properties. This uses proximity distances of the variables (as encountered in the individual cases) for clustering. In the case of dichotomous variables, the analysis standardizes the responses (by computing a mean and SD of the set of present/not present responses) and uses those standardized values as the location coordinates of the individual criteria. Once the distances are determined, the pair of criteria with the smallest distance are "clustered" and the centroid of that cluster $x a-x b$, $y a-y b$ is regarded as a "node" and the pair of criteria is no longer considered in distance computation. New distances are computed nodes and remaining criteria and the node establishment processes is repeated until all of the criteria (and nodes) are reduced to nodes in the displayed cluster hierarchy. Thus HCA provides a visual depiction of the relative relationships among criteria.

\section{RESULTS}

\section{GROUP CHARACTERISTICS}

There was no difference in the age of subjects between the SCA group ( $n=43 ; 58.3 \pm 14.6$ years $)$ and the psychogenic group $(n=40 ; 52.7 \pm 14.8$ years; $p>0.05)$. There was a significant difference $(p<0.001)$ in the number of criteria met between groups. Patients with SCA met an average of $2.2 \pm 1.4$ CDP criteria (range 0-6) while patients with previously identified psychogenic problems met an average of $4.1 \pm 1.7$ (range 1-7) CDP criteria. For patients with SCA, the most frequently met criteria were substandard performance on tests 1 and 2 and the calculated SOT score (Table 3). Note that four of the criteria were exhibited by two or fewer $(0-5 \%)$ of the SCA patients (lateral sway, regular periodicity of sway, circular sway, and inconsistent motor responses). Furthermore, only five patients with SCA met four or more criteria. For patients with known psychogenic problems, the most frequently met criteria were substandard performance on tests 1 and 2 and the calculated SOT score (Table 3 ). All other criteria were exhibited by from 3 to $28(7-70 \%)$ of the subjects. Twenty-five of the patients in the psychogenic group met four or more criteria.

\section{FREQUENCY DISTRIBUTIONS}

There were significant differences between the groups for the frequency of 3 of the 10 criteria used to identify psychogenic components to CDP (regular periodicity of sway, circular sway, and observed behaviors, Table 3 ). For each, the criteria occurred with

Table 3 | Frequency distribution of CDP criteria in patients with SCA and in patients with psychogenic problems.

\begin{tabular}{|c|c|c|c|}
\hline Criteria for psychogenic diagnosis & $\begin{array}{l}\% \text { Of SCA patients who } \\
\text { met each criteria }(n=43)\end{array}$ & $\begin{array}{l}\% \text { Psychogenic patients who } \\
\text { met each criteria }(n=40)\end{array}$ & $\begin{array}{l}\text { Chi-square level of } \\
\text { significance } p<0.005 \text { (2-sided) }\end{array}$ \\
\hline Calculated SOT score & $51(22)$ & $80(32)$ & 0.006 \\
\hline Substandard performance on sub-trials 1 and 2 & 77 (33) & $80(32)$ & .719 \\
\hline$>5^{\circ}$ AP sway on ST 4-6 without falls & $7(3)$ & $27.5(11)$ & .013 \\
\hline$>1.25^{\circ}$ lateral sway & $0(0)$ & $7.5(3)$ & .077 \\
\hline $\begin{array}{l}\text { Better on more difficult subtests than on easier } \\
\text { (ST } 5,6 \text { to ST 1, 2) }\end{array}$ & $7(3)$ & $15(6)$ & .255 \\
\hline Regular periodicity of sway & $4.7(2)$ & $35(14)$ & 0.001 \\
\hline Circular sway & $2(1)$ & $22.5(9)$ & .005 \\
\hline Increased inter-trial variability & $33(14)$ & $50(21)$ & .079 \\
\hline Inconsistent motor responses & $2(1)$ & $15.4(6)$ & 0.037 \\
\hline Observed behaviors (total) & $19(8)$ & $70(28)$ & 0.000 \\
\hline No. of Criteria met: $\leq 3$ of 10 & $88.4(38)$ & $37.4(15)$ & \\
\hline No. of Criteria met: $4-5$ of 10 & $9.3(4)$ & $42.5(17)$ & \\
\hline No. of Criteria met: $>5$ of 10 & $2.3(1)$ & $20(8)$ & \\
\hline
\end{tabular}


Table 4 | Sensitivity and specificity for each of the CDP and observational behaviors criteria.

\begin{tabular}{lccl}
\hline & $\begin{array}{l}\text { Sensitivity } \\
\text { (\%) }\end{array}$ & $\begin{array}{l}\text { Specificity } \\
(\mathbf{\%})\end{array}$ & $\begin{array}{l}\text { Positive } \\
\text { likelihood } \\
\text { ratio }\end{array}$ \\
& & & 1.56 \\
\hline Calculated SOT score & 80.0 & 48.8 & 1.04 \\
Substandard ST 1, 2 & 80.0 & 23.3 & 3.94 \\
$>5^{\circ}$ AP sway & 27.5 & 93.0 & - \\
$>$ 1.25 Lat sway & 7.5 & 100.0 & 2.15 \\
Better on ST 5, 6 to ST 1, 2 & 15.0 & 93.0 & 7.53 \\
Regular periodicity of sway & 35.0 & 95.2 & 9.67 \\
Circular sway & 22.5 & 97.6 & 1.61 \\
Increased inter-trial variability & 52.5 & 67.4 & 6.45 \\
Inconsistent motor & 15.4 & 97.6 & 3.76 \\
Observed behaviors & 70.0 & 81.4 & 0.42 \\
No. of Criteria met: $\leq 3$ of 10 & 37.5 & 11.6 & 3.66 \\
No. of Criteria met: 4-5 of 10 & 42.5 & 88.4 & - \\
No. of Criteria met: $>5$ of 10 & 20.0 & 100 & \\
\hline
\end{tabular}

a greater frequency in the psychogenic group. One additional criterion, Calculated SOT score, reached a near significant difference in frequency between the groups $(p<0.006)$.

\section{Sensitivity and specificity}

In general, each individual criterion had poor to good sensitivity (Table 4). That is, the proportion individuals with a diagnosis of PSY, based on the gold standard (neurologist's clinical impression), that are identified as PSY based on each criterion is low. However, the specificity of some of the criteria was good to excellent at correctly identifying people who are not in the psychogenic group. Those criteria with the best specificity (lateral sway, regular periodicity of sway, circular sway, and inconsistent motor) are those in which fewer than three of the SCA group presented with that criterion. These findings are supported by the highest positive likelihood ratios (scores $>1$ indicates that the test results is associated with psychogenic). Note that the positive likelihood ratio for lateral sway could not be calculated.

\section{PROXIMITIES}

Different patterns of relationships emerged for the SCA and the psychogenic groups, based on cluster analysis (Figure 1). For the SCA group, there is one very clear grouping in the criteria. This group has six of the criteria and represents the less frequently occurring criteria: large lateral sway, Inconsistent motor responses, circular sway, regular periodicity of sway, large AP sway, and better of ST 5, 6 than on ST 1, 2 .

For the psychogenic group, there is also one distinct grouping but the criteria represent the most frequently occurring criteria and have three criteria: Calculated SOT score, substandard performance on ST 1 and 2, and observed behaviors.

\section{DISCUSSION}

Computerized dynamic posturography criteria for psychogenic components: For over a decade, the results of CDP have been used to identify patients with a psychogenic component to their imbalance. In 1995, Cevette et al. (1995) compared the test results of patients with clinically identified "aphysiological" balance problems, patients with vestibular disorders and healthy subjects. Using step-wise linear discriminant analyses, they developed formulae to categorize patients as either "aphysiologic," "vestibular," or "normal" based on the raw data scores for peak-to-peak AP sway of the six sensory tests of CDP. In their hands, this formula accurately identified 95\% of the subjects considered to be "aphysiologic." Cevette et al. (1995) also identified three other criteria that could be used to identify an "aphysiological" component to balance performance: inter-trial variability across all trials, better performance on the more difficult tests (ST 5 and 6) than on the easiest tests (ST 1 and 2) based on a comparison of the average of the trials for a patient with the known reference values from healthy subjects. Goebel et al. (1997) added several other criteria including large amplitude AP sway $\left(>7.5^{\circ}\right)$, large amplitude lateral sway $\left(>2.5^{\circ}\right)$, exaggerated motor responses to small translational perturbations of the support surface and inconsistent motor responses to small and large translational perturbations of the support surface. They also considered substandard performance on ST 1, excessive intertrial variability but on ST 1 and 2 only, and better performance on ST 5 and 6 than on ST 1 and 2 although they used the best score in their calculations. The results of the study identified substandard performance on ST 1, and exaggerated motor responses and inconsistent motor responses to be best at identifying aphysiological balance performance. They suggested that the failure of large amplitude AP and lateral sway to identify subjects with an aphysiological component was because they set the criteria $\left(>7.5^{\circ}\right.$ and $>2.5^{\circ}$ respectively) too high. Krempl and Dobie (1998) compared the criteria suggested by Cevette et al. (1995) and by Goebel et al. (1997) using healthy subjects who performed as normal subjects, feigned malingering and performed under an induced vestibular dysfunction. Their results suggested that the best criteria were exaggerated motor responses to small perturbations of the support surface, inconsistent motor responses to small and large perturbations of the support surface and inter-trial variability.

Although no studies have applied the criteria established for psychogenic performance on CDP testing to patients with SCA, there is some evidence from previous studies that patients with SCA may meet at least some of the criteria. Increased sway when standing on a firm surface with eyes open and eyes closed (Substandard performance on ST 1 and/or 2) has been measured in patients with anterior lobe atrophy of the cerebellum, with olivo-ponto-cerebellar atrophy, with SCA, with cerebellar tumors and with cerebellar vermis lesions (Diener et al., 1984; Gill-Body et al., 1997; Baloh et al., 1998; Anderson et al., 2002). Studies of patients with chronic alcoholism also demonstrate increased sway on tests that parallel ST 1 and 2 (Sullivan et al., 2010). Other studies have mentioned large amplitude AP sway, abnormal medio-lateral sway and increased variability in sway of different sensory test conditions using CDP but do not provide the details that would enable the reader to extrapolate those findings to the criteria for psychogenic CDP (Ledin and Odkvist, 1991; Gill-Body et al., 1997; Sriranjini et al., 2009) Timmann and Horak (1998) reported that patients with cerebellar dysfunction had exaggerated sway following support surface perturbations, a criteria we 


\section{A}

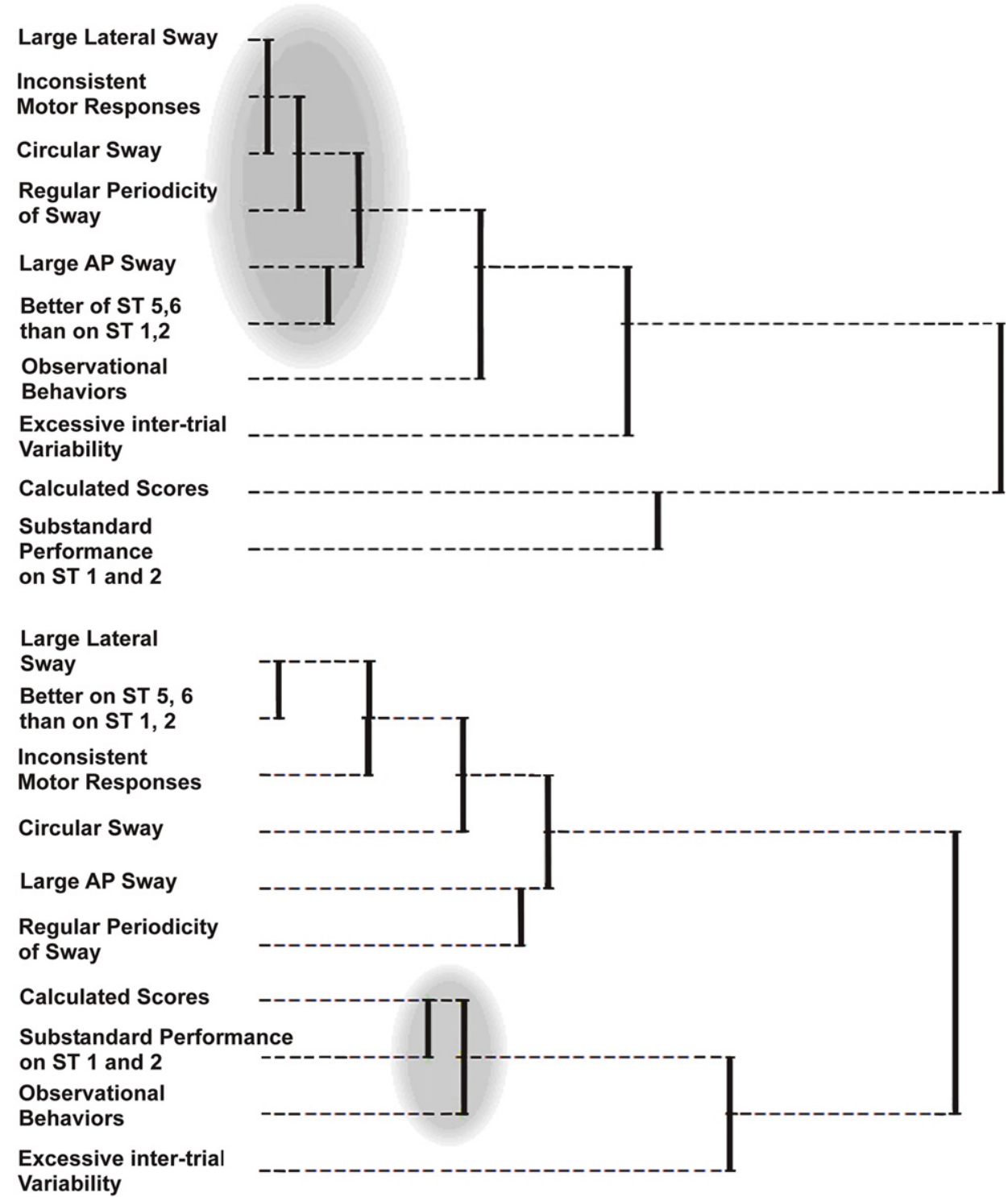

FIGURE 1 | Hierarchical cluster analysis (HCA) was used to organize the criteria into groups with similar properties. In HCA, relative distance of the point at which the nodes of the clusters combine is scaled from left to right. Thus the shorter the distances from left to right between two nodes, the closer the relationship between the criteria. (A) The dendrogram for the HCA of the SCA group identified one very clear grouping consisting of six criteria representing the less frequently occurring criteria (shaded area). (B) The dendrogram for the psychogenic group identified a clear grouping of three criteria representing the most frequently occurring criteria (shaded area). In addition, a cluster was formed between large lateral sway and better on ST 5, 6 than on 1,2 and another cluster consisting of large AP sway and regular periodicity of sway. chose not to use because we anticipated that patients with SCA would have exaggerated responses to perturbations. Several studies have described a high frequency AP sway in patients with cerebellar dysfunction (Diener et al., 1984; Wessel et al., 1988; Baloh et al., 1998). However, this postural tremor, associated with atrophy of the anterior lobe of the cerebellum, is of a higher frequency $(1-3 \mathrm{~Hz})$ than the regular periodicity of sway described as a psychogenic problem.

Individual CDP criteria were not particularly sensitive (identifying someone as PSY when they really were PSY) probably because the criteria look at only one potential contribution to
PSY and there may be many reasons for someone to be in the PSY group. Based on high positive likelihood ratios, which is calculated from both sensitivity and specificity, three criteria (regular periodicity of sway, circular sway, and inconsistent motor) appear to be associated with the PSY group. Large lateral sway would also belong in this group although positive likelihood ratio could not be calculated because specificity was $100 \%$. The positive likelihood ratios, however, indicates that if someone in the SCA group had the criteria, they belong in the PSY group.

The number of criteria met by each individual appears to be a useful way of categorizing people as belonging in the PSY group. 
Based on the positive likelihood ratios, our results were similar to those of Mallinson and Longridge (2005). That is, participants meeting three or fewer criteria were unlikely to have a psychogenic component to their balance problem, participants meeting four or five criteria possibly had a psychogenic component and participants meeting more than five criteria were likely to have a psychogenic component. These criteria for classifying patients as having or not having a psychogenic component need to be tested prospectively.

\section{LIMITATIONS}

Several aspects of this study are potential biases in the data. First, the diagnosis of psychogenic was based on the neurologist's clinical impression rather than on specific diagnostic tests. This may have resulted in some patients being categorized as having a psychogenic component to their dizziness when in fact they did not (false positives). Second, the technician may have known that a patient was considered to have a psychogenic component or had SCA prior to testing. An effort is made to avoid this bias by indicating to the technician that the patients referred for CDP testing have a diagnosis of "imbalance" rather than a more specific diagnosis. However, it remains possible that the technician would have additional information. Third, some of the criteria for a psychogenic component on CDP testing, specifically regular periodicity of sway and high inter-trial variability are based on qualitative judgment by the person interpreting the test results.

\section{REFERENCES}

Anderson, J. H., Christova, P. S., Xie, T., Schott, K. S., Ward, K., and Gomez, C. M. (2002). Spinocerebellar ataxia in monozygotic twins. Arch. Neurol. 59, 1945-1951.

Baloh, R. W., Jacobson, K. M., Beykirch, M. S., and Honrubia, V. (1998). Static and dynamic posturography on patients with vestibular and cerebellar lesions. Arch. Neurol. 55, 649-654.

Cevette, M. J., Puetz, B., Marion, M. S., Wertz, M. L., and Muenter, M. D. (1995). Aphysiologic performance on dynamic posturography. Otolaryngol. Head Neck Surg. 112, 676-688.

Cyr, D., and Cevette, M. J. (1993). "Classification of posturography patterns," in 20th Neurootological and Equilibriometric Society, Linkoping.

Diener, H. C., Dichgans, J., Bacher, M., and Gompf, B. (1984). Quantification of postural sway in normals and patients with cerebellar diseases. Electroencephalogr. Clin. Neurophysiol. 57, 134-142.

Gianoli, G., McWilliams, S., Soileau, J., and Belafsky, P. (2000). Posturographic performance in patients with the potential for secondary gain. Otolaryngol. Head Neck Surg. $122,11-18$.

Gill-Body, K. M., Popat, R. A., Parker, S. W., and Krebs, D. E. (1997). Rehabilitation of balance in two patients with cerebellar dysfunction. Phys. Ther. 77, 534-552.

Goebel, J. A., Sataloff, R. T., Hanson, J. M., Nashner, L. M., Hirshout, D. S., and Sokolow, C. C. (1997). Posturographic evidence of nonorganic sway patterns in normal subjects, patients, and suspected malingerers. Otolaryngol. Head Neck Surg. 117, 293-302.

Hall, C. D., and Herdman, S. H. (2005). "Dynamic posturography," in $\mathrm{Neu}$ rotology, eds R. K. Jackler and D. Brackmann (Orlando: Mosby, Inc.), 256-269.

Krempl, G. A., and Dobie, R. A. (1998). Evaluation of posturography in the detection of malingering subjects. Am. J. Otol. 19, 619-627.

Ledin, T., and Odkvist, L. M. (1991). Abstinent chronic alcoholics investigated by dynamic posturography, ocular smooth pursuit and visual suppression. Acta Otolaryngol. 111, 646-655.

Lempert, T., Brandt, T., Dieterich, M., and Huppert, D. (1991). How to identify psychogenic disorders of
Thus the test interpretation is subject to a "halo effect" where the presence of objectively determined psychogenic component (e.g., substandard performance of ST 1 and 2 or the calculated scores) might result in the addition of periodicity of sway and high intertrial variability to the components identified in the test results. Finally, the results of this study apply only to patients for who it is already known that their imbalance is related to either psychogenic problems or to SCA. Future studies are needed to determine the generalizability of our findings.

In summary, based on the combination of frequency of occurrence of different criteria, sensitivity and specificity, and positive likelihood ratios as well as and cluster analyses, we propose that the presence of certain criteria suggests a high likelihood that a patient has a psychogenic component to their balance impairment. Regular periodicity of sway across all trials, circular sway on SOT 4, 5, and 6, and inconsistent motor responses each had a high positive likelihood ratio reflecting the likelihood that if a person had these criteria, they should be categorized as having a psychogenic component to their balance problems. We would include large lateral sway as belonging in this group of criteria based on its high specificity. The absence of any or all of these criteria, however, would not mean that a patient does not have a psychogenic balance problem. Furthermore, the number of criteria met may also support categorizing someone as having a psychogenic component. Prospective studies are needed to validate these findings. stance and gait. A video study in 37 patients. J. Neurol. 238, 140-146.

Mallinson, A. L., and Longridge, N. S. (2005). A new set of criteria for evaluation malingering in workrelated vestibular injury. Otol. Neurotol. 26, 686-690.

Manto, M.-U. (2005). The wide spectrum of spinocerebellar ataxias (SCAs). Cerebellum 4, 2-6.

Sriranjini, S. J., Pal, P. K., Devidas, K. V., and Ganpathy, S. (2009). Improvement in balance in progressive degenerative cerebellar ataxias after Ayurvedic therapy: a preliminary report. Neurol. India 57, 116-171.

Sullivan,E.V.,Rise,J.,andPfefferbaum,A. (2010). Physiological and focalcerebellar substrates of abnormal postural sway and tremor in alcoholic women. Biol. Psychiatry 67, 44-51.

Teive, H. A. (2009). Spinocerebellar ataxias. Arq. Neuropsiquiatr. 67, 1133-1142.

Timmann, D., and Horak, F. B. (1998). Perturbed step initiation in cerebellar subjects. 1. Modifications of postural responses. Exp. Brain Res. 119, 73-84.

Wessel, K., Diener, H. C., Dichgans, J., and Thron, A. (1988). Cerebellar dysfunction in patients with bronchogenic carcinoma: clinical and posturographic findings. J. Neurol. 235, 290-296.

Conflict of Interest Statement: The authors declare that the research was conducted in the absence of any commercial or financial relationships that could be construed as a potential conflict of interest.

Received: 01 September 2010; accepted: 21 March 2011; published online: 04 April 2011.

Citation: Herdman SJ, Hall CD, Eggers $R$, Sampson S, Goodier $S$ and Filson $B$ (2011) Misclassification of patients with spinocerebellar ataxia as having psychogenic postural instability based on computerized dynamic posturography. Front. Neur. 2:21. doi: 10.3389/fneur.2011.00021

This article was submitted to Frontiers in Neuro-otology, a specialty of Frontiers in Neurology.

Copyright $\odot 2011$ Herdman, Hall, Eggers, Sampson, Goodier and Filson. This is an open-access article subject to a nonexclusive license between the authors and Frontiers Media SA, which permits use, distribution and reproduction in other forums, provided the original authors and source are credited and other Frontiers conditions are complied with. 